\title{
Unusual yet isolated oral manifestations of persistent thrombocytopenia-A rare case report
}

\author{
Amit Byatnal', Neha Mahajan², Shrinivas Koppal', A Ravikiran ${ }^{3}$, Thriveni R', Parvathi Devi M K ${ }^{1}$ \\ ${ }^{1}$ Department of Oral Medicine \& Radiology, AMES Dental College and Hospital, Raichur, Karanataka \\ ${ }^{2}$ Department of Oral Medicine \& Radiology, Private consultant Shimla, Himachal Pradesh \\ ${ }^{3}$ Department of Oral Medicine and Radiology, Sibar Institute of Dental Sciences, Guntur, Andhra Pradesh
}

\begin{abstract}
Patients with platelet-mediated disorders often present clinical manifestations of bruising and bleeding. Although these changes are detected most frequently on the skin, the oral cavity also may exhibit signs of bleeding. In this report, we describe a patient who presented isolated oral features of hemorrhagic bullae with bleeding, indicative of a bleeding disorder. Results of laboratory tests revealed severe thrombocytopenia and a careful history disclosed dengue fever as the cause. The importance of recognizing the oral manifestations of thrombocytopenia is highlighted here, since the oral cavity is a frequent site of hemorrhage and could be the only manifestation of the disease.
\end{abstract}

Keywords: thrombocytopenia, platelet disorder, dengue fever.

\section{Introduction}

Platelets or thrombocytes are a critical component of vascular 'plugs' that form during hemostasis to limit blood loss secondary to vascular damage. Consequently, disorders accompanied by insufficiencies of platelet number or platelet dysfunction may contribute to pathologic bleeding states ${ }^{1}$. A platelet count of $150-450 \times 10^{3} / \mathrm{mm}^{3}$ is considered normal ${ }^{2}$. Thrombocytopenia is a hematologic disorder that is characterized by a markedly decreased number of circulating blood platelets.

A reduction in platelet number or function can occur through a variety of mechanisms, including autoimmune destruction, spleen sequestration, bone marrow infiltration by tumor cells, infection (e.g. dengue fever), and adverse drug reaction. Regardless of the cause, platelet disorders typically manifest with petechiae, purpura, and bleeding of the mucous membranes ${ }^{3}$. Although these changes are detected most frequently on the skin; the oral cavity, the nasal and genital mucosa, as well as the renal and gastrointestinal systems, also may exhibit signs of bleeding ${ }^{4}$. Spontaneous clinical hemorrhages are usually not observed until platelet counts fall below $30 \times 10^{3} / \mathrm{mm}^{3}$.

Gingival bleeding, either spontaneous or in response to minor trauma (i.e. tooth brushing, flossing), is often the first sign of thrombocytopenia. The oral mucosa, most notably the soft palate and buccal mucosa, may demonstrate petechiae and ecchymoses. Deep red to black hemorrhagic bullae may occur with very low platelet counts ${ }^{3}$.

In case of dengue viral fever, mucocutaneous manifestations play significant 
role in its diagnosis. Though most of these manifestations are noted on ocular mucosa like conjunctiva, sclera, there have been reports of dengue fever presenting as small vesicles on soft palate, erythema and crusting of lips and tongue ${ }^{5}$.

Here we report a case of thrombocytopenia associated with dengue fever, which showed only oral manifestations, thus highlighting the significance of recognizing the oral signs.

\section{Case Report}

A 50-year-old female patient reported to the department of Oral Medicine and Radiology with a history of spontaneous and profuse bleeding of tongue for the last six months. Patient had suffered from dengue fever six months before, for which she had undergone blood transfusion, as per her previous medical reports. Patient was married for the past 20 years and had 2 children. Her family history and review of systems also did not reveal any significant findings.

Intraoral examination revealed the presence of multiple hemorrhagic bullae on left sublingual mucous membrane as well as left lateral surface of the tongue and floor of the mouth (Figure 1). Overlying surface was blue-black in color, and profuse bleeding occurred on slight palpation. Right side of palate and right posterior buccal mucosa revealed the presence of brown color plaques with rough surface. These lesions also showed bleeding on touch (Figures 2 and 3). Based on the clinical examination, hematological disorder due to dengue fever was chiefly considered, while differential diagnoses included oral squamous cell carcinoma of tongue and buccal mucosa and other infections like influenza, measles, rubella and bacterial sepsis. Hematological examinations revealed platelet level less than $40,000 / \mathrm{mm}^{3}$, along with decrease in level of hemoglobin, red blood cells count, packed cell volume and erythrocyte sedimentation rate.

Based on the history of dengue fever with the presence of oral manifestations of hemorrhagic bullae and a laboratory report confirming decreased platelet count, a final diagnosis of persistent thrombocytopenia after dengue fever was given. The patient was referred to general medicine department for further systemic treatment. But we lost the patient to follow up as she succumbed to her illness.

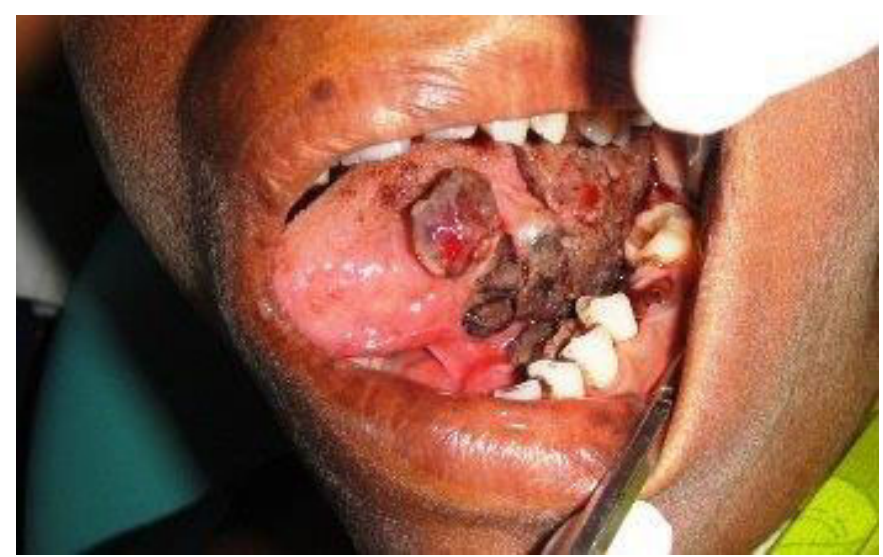

Fig. 1 - Multiple hemorrhagic bullae on left sublingual mucous membrane, left lateral border of tongue and floor of the mouth

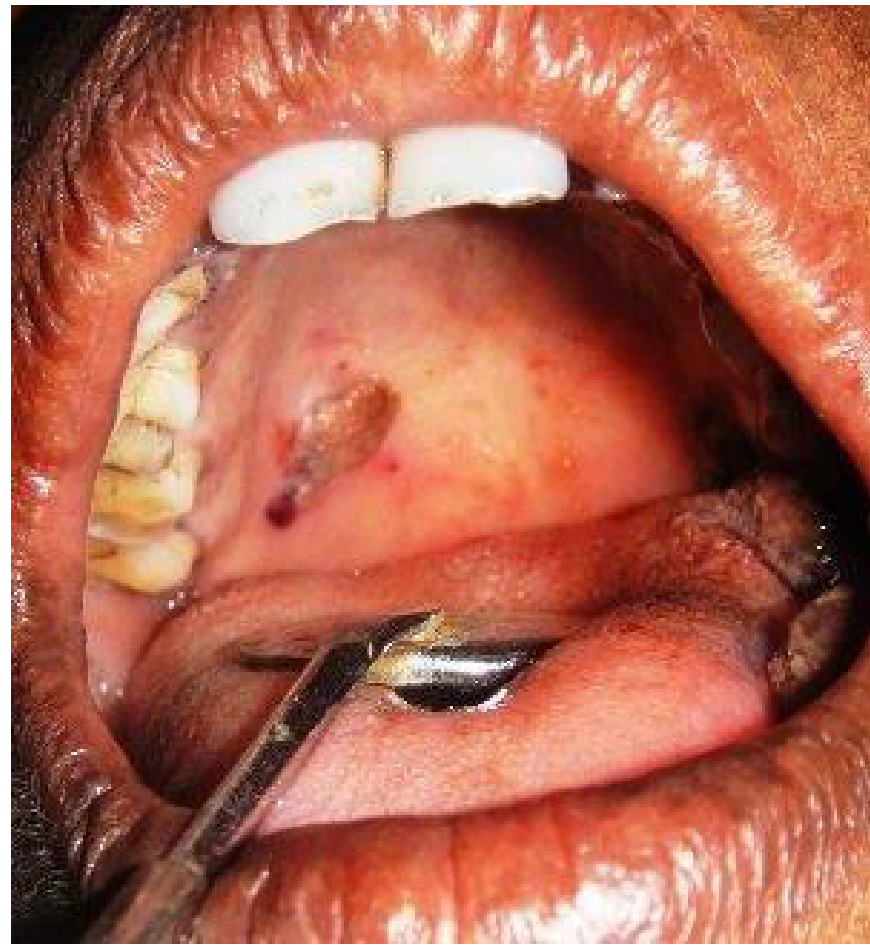

Fig. 2: Brown plaques on the right palatal surface

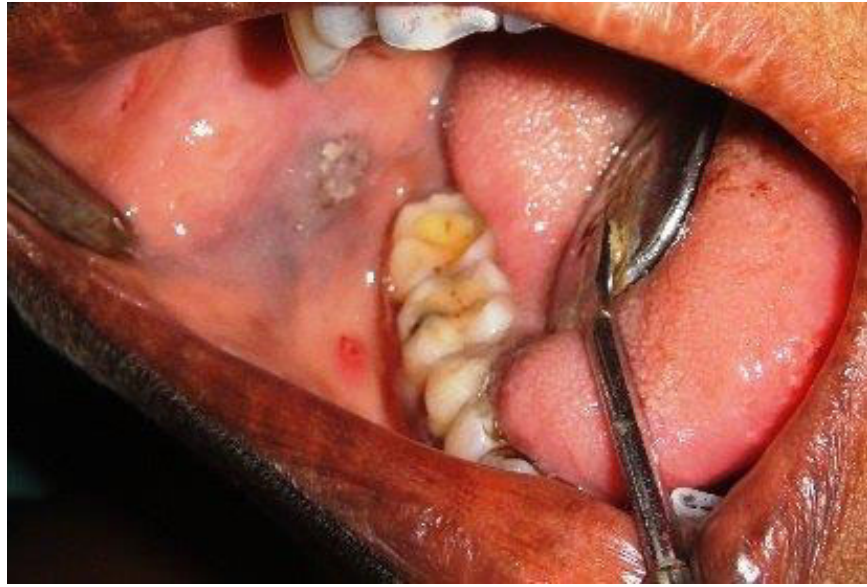

Fig. 3: Brown plaques on the right posterior buccal mucosa

\section{Discussion}

Dengue is probably the most imperative mosquito-borne viral disease in the world, in terms of morbidity and mortality. It is caused by dengue virus that belongs to flaviviridae group, while the vector is aedes aegypti mosquito that once infected remains infected for life and is able to transmit to susceptible individuals. Dengue affects tropical and subtropical regions very commonly ${ }^{6}$. Co-circulation of the four different types of dengue viruses and expansion of dengue epidemic has given rise to infection enhancement and a big expansion of clinical aspects of the disease ${ }^{7}$.

Dengue virus infections cause a spectrum of illness ranging from asymptomatic, mild undifferentiated fever to classical dengue fever (DF), and dengue fever with 
hemorrhagic manifestations, or dengue hemorrhagic fever (DHF) and the dengue shock syndrome (DSS). Each year an estimated 100 million cases of DF occur and between 250,000 and 500,000 cases of DHF are reported to $\mathrm{WHO}^{6}$. The SouthEast Asian countries such as India, Indonesia, Myanmar and Thailand are at the highest risk of DF/DHF, accounting for nearly half the global risk.

Criteria for diagnosis of dengue hemorrhagic fever include a probable or confirmed case of dengue infection while hemorrhagic tendencies are evidenced by one or more of the following: a positive result from tourniquet test; petechiae, ecchymoses or purpura; bleeding from mucosa, gastrointestinal tract, injection sites; hematemesis or melena; and thrombocytopenia $\left(<100,000 / \mathrm{mm}^{3}\right)$ with an evidence of plasma leakage due to increased vascular permeability ${ }^{8}$. In the present case, history of previously confirmed diagnosis of dengue infection along with oral mucosal lesions exhibiting bleeding tendency, in association with platelet count $<40000 / \mathrm{mm}^{3}$ pointed toward dengue hemorrhagic fever. As there were no other findings to fulfill the criteria of DHF, it is significant to consider dengue fever and thrombocytopenia as one of the prime diagnosis based on oral manifestations. Thrombocytopenia and elevated transaminases have been observed in patients with classic dengue fever ${ }^{9}$.

Cases of hemorrhagic bullae and other oral manifestations of thrombocytopenia have been reported ${ }^{10-12}$. Bleeding in dengue is one of the dreaded complications and is associated with higher mortality in dengue DHF/DSS. Bleeding manifestations are highly variable and do not always correlate with the laboratory abnormalities in the coagulation profile. Factors like mild degree of disseminated intravascular coagulation (DIC), hepatic derangement and thrombocytopenia act synergistically to cause bleeding in dengue patient ${ }^{13}$. Severe bleeding is related to severe thrombocytopenia ${ }^{14}$.

Hemorrhagic manifestations include skin hemorrhages in the form of petechiae, purpurae, ecchymoses; gingival bleeding, nasal bleeding, gastrointestinal bleeding in form of hematemesis, melena, hematochezia; hematuria and increased menstrual flow. Up to half of patients with dengue fever develop a characteristic rash, which is variable and may be maculopapular or macular, and, petechiae and purpura develop as hemorrhagic manifestations, commonly at venepuncture sites.

An aberrant immune over activation after dengue virus infection not only impairs the immune response to clear the virus, but also results in overproduction of cytokines that affect monocytes, endothelial cells and hepatocytes, as well as the abnormal production of autoantibodies to platelets and endothelial cells. A molecular mimicry occurs between platelets or endothelial cells and dengue virus antigens. Dengue virus-induced vasculopathy and coagulopathy are involved in the pathogenesis of hemorrhage ${ }^{15}$.

Vaughn et al. ${ }^{16}$ demonstrated the correlation of virus serotype with disease severity in pediatric patients, but there are no available data regarding this correlation in the adult population.

Differential diagnosis of acquired thrombocytopenia includes disseminated intravascular coagulation, thrombotic thrombocytopenic purpura, drug induced post-transfusion purpura and idiopathic or immune mediated thrombocytopenic purpura (ITP $)^{12}$.

Dengue fever is usually a self-limited illness with no specific currently available antiviral treatment. The World Health Organization (WHO) has provided guidelines for treatment of dengue fever/dengue hemorrhagic fever, which included supportive care with analgesics, fluid replacement and bed rest. However, in the present case the patient unfortunately succumbed to her illness before the treatment could be initiated.

The two clinical tests used to evaluate primary hemostasis are the platelet count and bleeding time (BT). As platelet count reduces in patients with thrombocytopenia, bleeding time increases. BT is usually considered to be normal between 1 and 6 minutes (by modified Ivy's test) and is prolonged when greater than 15 minutes. Prothrombin time (PT) or international normalized ratio (INR) and activated partial thromboplastin time (aPTT) remains normal ${ }^{17}$.

Patients with platelet counts greater than $30 \times 10^{3} / \mathrm{mm}^{3}$ require no treatment unless they are about to undergo a surgical procedure. First-line therapy consists of oral corticosteroids $1 \mathrm{mg} / \mathrm{kg}$ body weight. Second-line therapy involves splenectomy, to which the majority of patients respond. Other treatments include high-dose intravenous (i.v.) $\mathrm{IgG}$, i.v. anti-D, vinca alkaloids, danazol and immunosuppressive agents such as azathioprine, cyclosporine and dapsone. There is also interest in the use of specific monoclonal antibodies such as rituximab, as well as recombinant thrombopoietin.

Platelet transfusions are reserved for intracranial or other extreme hemorrhage, where emergency splenectomy may be justified. Transfusion of 6 to 10 units of platelets will increase patient platelet counts by 17 to $31 \times 10^{3} / \mathrm{mm}^{3}$, respectively. With advances in apheresis technology, a single donor in one apheresis sitting can produce 6 to 10 units of leukocytereduced platelets ( 3 to $8 \times 10^{11}$ platelets). As a consequence, single-donor infusions can be used in place of platelets pooled from multiple donors. The advantages of this practice are reduced donor exposure, lower incidence of alloimmunization and lower refractoriness to platelet transfusions ${ }^{18}$.

Dengue should hence be suspected in patients with hematological disorders and autoimmune features in endemic regions ${ }^{7}$. It is also important to recognize the oral manifestations of thrombocytopenia since the oral cavity is a frequent site of hemorrhage and may be the only manifestation of the disease. Proper identification permits the prompt institution of treatment and prevention of serious complications.

\section{References}

1. Cleveland DB, Rinaggio J. Oral and maxillofacial manifestations of systemic and generalized disease. Endodontic Topics. 2003; 4: 69-90.

2. Nicki RC, Brian RW, Stuart HR, editors. Davidson's principles and practice of medicine. 21th ed. Churchill Living Stone Elsevier; 2010.

3. Schlosser BJ, Pirigyi M, Mirowski GW. Oral manifestations of hematologic and nutritional diseases. Otolaryngol Clin N Am. 2011;44: 183-203. 
4. Eisen D, Essell J. Drug-induced thrombocytopenia presenting with isolated oral lesions: report of two cases. Cutis. 1998; 62: 193-5.

5. Thomas EA, John M, Kanish B. Mucocutaneous manifestations of dengue fever. Indian J Dermatol. 2010; 55: 79-85.

6. World Health Organization: Dengue and dengue haemorrhagic fever. Factsheet n.117. Genebra: WHO; 2002.

7. Jardim DL, Tsukumo DM, Angerami RN, Carvalho Filho MA, Saad MJ. Autoimmune features caused by dengue fever: a case report. Braz $\mathrm{J}$ Infect Dis. 2012; 16: 92-5.

8. Chuansumrit $A$, Tangnararatchakit K. Pathophysiology and management of dengue haemorrhagic fever. Transfus Altern Transfus Med. 2006; 8: 3-11.

9. Dengue: profile of haematological and biochemical dynamics. Rev Bras Hematol Hemoter. 2012; 34: 36-41.

10. Eisen D, Essell J. Drug-induced thrombocytopenia presenting with isolated oral lesions: report of two cases. Cutis. 1998; 62: 193-5.

11. James WD, Guiry CC, Grote WR. Acute idiopathic thrombocytopenic purpura. Oral Surg Oral Med Oral Pathol. 1984; 57: 149-51.

12. Helms AE, Schaffer RI. Idiopathic thrombocytopenic purpura with black oral mucosal lesions. Cutis. 2007; 79: 456-8.

13. Shivbalan S, Anandnathan K, Balasubramanian S, Datta M, Amalraj E. Predictors of spontaneous bleeding in Dengue. Indian J Pediatr. 2004; 71: 33-6.

14. Chairulfatah A, Setiabudi D, Agoes R, Colebunder R. Thrombocytopenia and platelet transfusions in dengue Haemorrhagic Fever and Dengue Shock Syndrome. WHO Dengue Bulletin. 2003; 27: 141-3.

15. Lei HY, Huang KJ, Lin YS. Immunopathogenesis of dengue haemorrhagic fever. Am J Infect Dis. 2008; 4: 1-9.

16. Vaughn DW, Green S, Kalayanarooj S, Innis BL, Nimmannitya S, Suntayakorn $S$ et al. Dengue viremia titer, antibody response pattern and virus serotype correlate with disease severity. J Infect Dis. 2000; 181: 2-9.

17. Greenberg MS, Glick M, editors. Burket's oral medicine: diagnosis and treatment. 11th ed. Lewiston, NY: B.C. Decker; 2008.

18. Drews RE. Critical issues in haematology: anaemia, thrombocytopenia, coagulopathy, and blood product transfusions in critically ill patients. Clin Chest Med. 2003; 24: 607-22. 